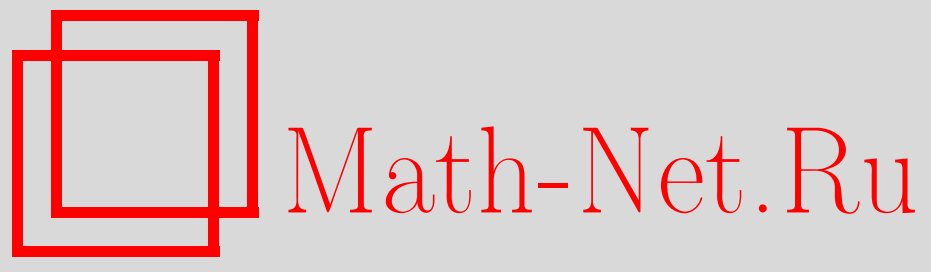

А. Е. Миронов, Коммутирующие разностные операторы с полиномиальными коэффициентами, УМН, 2007, том 62, выпуск 4, 169-170

DOI: https://doi.org/10.4213/rm6848

Использование Общероссийского математического портала Math-Net.Ru подразумевает, что вы прочитали и согласны с пользовательским соглашением http://www . mathnet.ru/rus/agreement

Параметры загрузки:

IP: 3.95 .254 .165

26 апреля 2023 г., 07:21:49

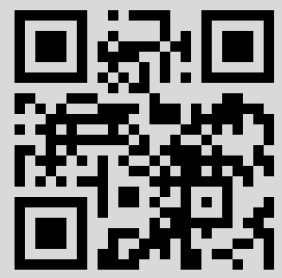




\section{Коммутирующие разностные операторы с полиномиальными коэффициентами}

\section{А. Е. Миронов}

В этой работе в рамках одноточечной конструкции найдены коммутирующие разностные операторы ранга 2 , отвечающие кривой рода 1 , коэффициенты которых являются полиномами от дискретной переменной.

Совместные собственные функции коммутирующих разностных операторов

$$
L_{1}=\sum_{N_{-}}^{N_{+}} u_{i}(n) T^{i}, \quad L_{2}=\sum_{M_{-}}^{M_{+}} v_{i}(n) T^{i},
$$

где $T$ - оператор сдвига по дискретной переменной $n \in \mathbb{Z}$, параметризуются спектральной кривой $\Gamma$, заданной в $\mathbb{C}^{2}$ некоторым полиномом $Q(\lambda, \mu)$ :

$$
L_{1} \psi(n, P)=\lambda \psi(n, P), \quad L_{2} \psi(n, P)=\mu \psi(n, P), \quad P=(\lambda, \mu) \in \Gamma .
$$

Рангом $l$ пары операторов $L_{1}, L_{2}$ называется размерность пространства совместных собственных функций в точке $P \in \Gamma$ общего положения. При $l=1$ функция $\psi(n, P)$ выражается через тета-функцию кривой $\Gamma$ и коэффициенты операторов легко восстанавливаются [1]. При $l>1$ нахождение функции $\psi(n, P)$ сводится к решению задачи Римана и найти эту функцию в явном виде не удается. И. М. Кричевером и С. П. Новиковым [1] (см. также [2]) предложен метод отыскания коэффициентов операторов, называемый методом деформации параметров Тюрина, который не требует нахождения $\psi(n, P)$. Ими показано, что коэффициенты операторов можно восстановить из решений уравнений на параметры Тюрина голоморфных стабильных расслоений, которые однозначно задаются функцией $\psi(n, P)$, при этом коэффициенты операторов зависят от произвольных $l$ функциональных параметров. А именно, ими показано, что для восстановления коэффициентов операторов достаточно найти матричную функцию

$$
\chi(n, P)=\Psi(n+1, P) \Psi^{-1}(n, P),
$$

где $\Psi(n, P)$ - матрица Вронского, построенная по некоторому базису в пространстве совместных собственных функций. Используя метод деформации параметров Тюрина, Кричевер и Новиков нашли операторы ранга 2, отвечающие эллиптической кривой. При этом коэффициенты операторов выражены через $\zeta$ - и $\wp$-функции Вейерштрасса от двух функциональных параметров.

В этой работе указаны спектральные данные для операторов ранга 2, отвечающих кривой рода 1, коэффициенты которых выражаются через элементарные функции от функциональных параметров. В частности, мы укажем операторы, которые, как и их гладкие аналоги - операторы Диксмье [3], имеют полиномиальные коэффициенты.

Пусть кривая Г задана в $\mathbb{C}^{2}$ уравнением

$$
w^{2}=F(z)=z^{4}+c_{2} z^{2}+c_{1} z+1 .
$$

Кривая Г допускает голоморфную инволюцию $\sigma: \Gamma \rightarrow \Gamma, \sigma(z, w)=(z,-w)$. При $l=2$ матрица $\chi(n, P)$ имеет вид

$$
\chi(n, P)=\left(\begin{array}{cc}
0 & 1 \\
\chi_{1}(n, P) & \chi_{2}(n, P)
\end{array}\right), \quad P=(z, w) \in \Gamma .
$$

Работа выполнена при поддержке РФФИ (грант 06-01-00094a), гранта Президента Российской Федерации MK-9651.2006.1 и Фонда содействия отечественной науке. 
Мы рассматриваем случай, когда инволюция $\sigma$ не изменяет $\chi_{1}$, т. е.

$$
\chi_{1}(n, P)=\chi_{1}(n, \sigma(P)) .
$$

Tеорема 1. Функиии $\chi_{1}(x, P)$ и $\chi_{2}(x, P)$ имеют вид

$$
\chi_{1}=\frac{c(n)}{z-\gamma(n)}+\frac{c(n)}{\gamma(n)-\gamma(n+1)}, \quad \chi_{2}=\frac{1}{2 z}+\frac{a(n)}{2(z-\gamma(n))}+\frac{w \gamma(n)}{2 z(\gamma(n)-z)}+d(n),
$$

где

$c(n)=\frac{\gamma(n-1)\left(a^{2}(n)-F(\gamma(n))\right)}{4 \gamma(n)(\gamma(n)-\gamma(n-1))}, \quad d(n)=\frac{(a(n+1)-1) \gamma(n)+(a(n)+1) \gamma(n+1)}{2(\gamma(n)-\gamma(n+1)) \gamma(n+1)}$, $\gamma(n), a(n)$ - произвольные функции дискретной переменной $n \in \mathbb{Z}$.

Теорема 2. При $a(n)=n+1, \gamma(n)=n$ операторы имеют полиномиальные по $n$ коэффициенты. Оператор второго порлдка имеет вид

$$
\begin{aligned}
L_{2}=T^{2} & +2(n+2) T-\left(\frac{n^{4}}{2}+n^{3}-\frac{1}{2}\left(1-c_{2}\right) n^{2}-\frac{1}{2}\left(8-c_{1}-c_{2}\right) n\right) \\
& -\frac{1}{2}\left(n^{3}+\left(c_{2}-1\right) n+c_{1}-2\right)\left(n^{2}+n-1\right) T^{-1} \\
& +\frac{1}{16}\left(n^{3}+\left(c_{2}-1\right) n+c_{1}-2\right)\left(n^{3}-3 n^{2}+\left(2+c_{2}\right) n+c_{1}-c_{2}-2\right) \\
& \times(n+1)(n-2) T^{-2} .
\end{aligned}
$$

Отметим, что аналог редукции (1) в гладком случае мы использовали в [4] для нахождения коммутирующих дифференциальных операторов ранга 2, рода 2.

\section{Список литературы}

[1] И. М. Кричевер, С.П. Новиков, Функи. анализ и его прилож., 12:4 (1978), 41-52. [2] И. М. Кричевер, С. П. Новиков, УМН, 58:3 (2003), 51-88. [3] Ж. Диксмье, Математика, 13:4 (1969), 16-44. [4] А. Е. Миронов, Матем. сб., 195:5 (2004), 103-114.

\section{А. Е. Миронов (А. Е. Mironov)}

Институт математики им. С. Л. Соболева СО РАН, Новосибирск;

Новосибирский государственный университет

E-mail: mironov@math.nsc.ru
Представлено И. М. Кричевером Принято редколлегией 27.04 .2007 